\title{
NETWORKED ROBUST PREDICTIVE CONTROL SYSTEMS DESIGN WITH PACKET LOSS
}

\author{
Quang T. Nguyen* _ Vojtech Veselý ${ }^{* *}$ \\ Alena Kozáková ${ }^{* *}$ _ Pavel Pakshin ${ }^{* * *}$
}

\begin{abstract}
The paper addresses problem of designing a robust output feedback model predictive control for uncertain linear systems over networks with packet-loss. The packet-loss process is arbitrary and bounded by the control horizon of model predictive control. Networked predictive control systems with packet loss are modeled as switched linear systems. This enables us to apply the theory of switched systems to establish the stability condition. The stabilizing controller design is based on sufficient robust stability conditions formulated as a solution of bilinear matrix inequality. Finally, a benchmark numerical example-double integrator is given to illustrate the effectiveness of the proposed method.
\end{abstract}

K e y w ords: model predictive control (MPC), networked control systems (NCSs), packet-loss, parameter dependent quadratic stability (PDQS), bilinear matrix inequality (BMI)

\section{INTRODUCTION}

Feedback control systems wherein the loops are closed through real-time networks are called Networked Control Systems (NCSs) [16, 18, 26, 29]. Advantages of using NCSs in the control area include simplicity, costeffectiveness, ease of system diagnosis and maintenance, increased system agility and testability. However, the integration of communication real-time networks into feedback control loops inevitable leads to challenging problems such as network-induced delays and data packet losses, which can induce instability or poor performance of closed-loop control systems. Therefore, packet loss is one of the most important and special issues of NCSs.

There are two major approaches to accommodate the issue of packet loss in an NCS design. One way is that one first designs the control system without regard to the networks, and then determines a performance level that the networks should satisfy (for example, maximum allowable transfer interval) so that the closed-loop system maintains its performance (for example, stability) when some control and sensor signals are transmitted via the networks $[13,29]$. The other approach is to treat the network protocol and traffic as given conditions and designs the control strategies that explicitly take the networkinduced issues into account $[1,21,27,28]$. Under the assumption that the network is modeled as a switch governed by a Bernoulli process, Zhang et al [29] proposed a criterion to check whether the NCS is stable at a certain rate of packet losses, and searched for the maximum packet-loss rate under which the overall system remains stable. The method they used derives from the stability analysis for asynchronous dynamic systems. With packetloss rate known and constant, Seiler and Sengupta [21] formulated the NCS as a Markovian jump system with two operation modes, and then applied the techniques developed for Markovian jump systems. A dynamic output feedback controller design method was proposed such that the NCS is mean square stable and has $H_{\infty}$ gain below certain value in terms of linear matrix inequalities (LMIs). Moreover, Yu et al [28] modeled the packet-loss process as an arbitrary but finite switching signal. This enables them to apply the theory from switched systems to stabilize the NCS. However, in the framework considered in the references mentioned above, the controller is directly connected to the actuator. That means no packets are dropped in control signals. A general framework was considered in [1], where both sampling signals and control commands are transmitted through the network and may be dropped during the transmissions. The linear quadratic Gaussian control problem was studied based on dynamic programming approach. Xiong and Lam [27] generalized the procedure in [28] to double-sided packet loss, as one of the contributions, and established stability conditions via a packet-loss dependent Lyapunov approach, as another contribution.

In the last two decades, model predictive control (MPC) has been widely adopted in industry as an effective means to deal with multivariable constrained control problems $[8,17]$. The idea of MPC is stemmed from employing an explicit model of the plant to be controlled which is used to predict the future state/output behaviour over the finite time horizon. There are some researching results that have been presented in MPC for NCSs. Srinivasaguta et al [22] proposed a time-stamped model predictive control algorithm for NCS when random delay is less than one sample time. Liu et al [10] proposed using a networked control predictor to take the

\footnotetext{
* Samsung Electronics Slovakia, Research and Development, Circuit Group, q.nguyen@samsung.com, ${ }^{* *}$ Slovak University of Technology, Faculty of Electrical Engineering and Information, Technology, Institute of Control and Industrial Informatics, Ilkovičova 3, 81219 Bratislava, Slovakia, vojtech.vesely@stuba.sk, alena.kozakova@stuba.sk, *** Arzamas Polytechnic Institute of R.E.Alekseev, Nizhny Novgorod State Technical University, Russia, pakshinpv@gmail.com

A short version of this paper has been published at 4th IFAC Symposium NMPC'12
} 
latest control value from the predictive control sequence available to deal with random communication time delay problem, and then $\mathrm{Mu}$ et al [12] presented using a low pass filter to filter the error produced between the delayed plant output measurement and its delayed openloop model output to improve system robustness. Tang and de Silva [23] proposed a NCS control strategy based on generalized predictive control with the buffering of future control sequence to overcome the transmission delay problems at the controller-to-actuator lines and Tang and de Silva [23] presented the conditions under which the stability of the constrained model predictive control for NCS with random delay can be guaranteed. Li et al [9] proposed a stabilizing MPC strategy for NCS with data packet loss between sensor and controller. In [2] the authors presented a packet-based robust MPC approach in a co-design framework for Wireless Networked Control Systems (WNCS) with the round-trip delay. Polytopic description was used to describe uncertainty of system. In $[3,4]$ the author proposed an MPC design method for NCS with double-sided packet loss. A packet-loss dependent Lyapunov function is used for stabilization, and the result is used for synthesizing model predictive control by parameterizing the infinite horizon control moves into a single state feedback law. However, Ding [3] assumes that the actuator information (whether or not the actuator has received new data) can be sent to the controller and in [4] he investigates the case when the actuator information cannot be sent to the controller. One of the ways to overcome the resulting loss-packet problems is the use of prediction based compensation schemes $[2,5-7,14]$. For example, in [6], the authors presented a networked control scheme which uses a model based prediction and time-stamps in order to compensate for delays and packet dropouts in the transmission between sensor and controller and between controller and actuator, respectively. In order to analyze the properties of the scheme, they introduced the notion of prediction consistency which enables them to precisely state the network properties needed in order to ensure stability of the closed loop.

From our vision, it seems that there is no previous result on design of robust output feedback MPC for uncertain linear systems over networks with bounded packet loss. Motivated by the above observation, in this paper, we consider the implementation of a robust output feedback linear model predictive control scheme over a network with double-sided packet loss. The main idea of this paper is based on the combination of compensation mechanism in $([2,5-7]$ and robust model predictive control design approach in [15]. As a result, networked predictive control systems with loss packet are modeled as switched linear systems. This enables us to apply the theory of switched systems to establish the stability condition of networked model predictive control. We suppose that network-induced delays are within the sample time. Packet loss process is considered as arbitrary but bounded by the horizon control of MPC and its contribution is based on [27]. The main goal of this paper is to provide a robust stability analysis and synthesis robust predictive controller for this scheme with guaranteed cost and PDQS.

The organization of the paper is as follows. Section 2 gives the problem formulation. In Section 3, the robust output feedback predictive controller design method with input constraints using bilinear matrix inequality is presented. The approach of robust constrained networked model predictive control design with guaranteed cost and PDQS is introduced in section 4. In section 5, one benchmark example is solved by using Yalmip BMI solvers to show the effectiveness of the proposed method. Finally, some conclusions are given.

Hereafter, the following notational conventions will be adopted: given a symmetric matrix $P=P^{T}$, the inequality $P>0(P \geq 0)$ denotes matrix positive definiteness (semi-definiteness). "*" denotes a block that is transposed and complex conjugate to the respective symmetrically placed one. Matrices, if not explicitly stated, are assumed to have compatible dimensions. I denotes the identity matrix of corresponding dimensions. The notation $x(t+k \mid t)$ will be used to define, at time $t k$ - steps ahead, prediction of a system variable $x$ from time $t$ onwards under a specified initial state and input scenario. Note that $x(t \mid t)=x(t)$.

\section{PRELIMINARIES AND PROBLEM FORMULATION}

The framework of NCS considered in the paper is depicted in Fig. 1. Let the polytopic model of the plant to be controlled be described by the following linear discrete time difference equation

$$
\begin{aligned}
x(t+1) & =A(\xi) x(t)+B(\xi) u(t), \\
y(t) & =C x(t)
\end{aligned}
$$

where $x(t) \in R^{n}, u(t) \in R^{m}$, and $y(t) \in R^{l}$ denote the state, control input, and output respectively. The matrices $A(\xi), B(\xi)$ belong to convex and bounded set $S$, which is a polytop with $N$ vertices $S_{1}, S_{2}, \ldots, S_{N}$ that can be formally defined as follows

$$
\begin{gathered}
S:=\left\{A(\xi) \in R^{n \times n}, B(\xi) \in R^{n \times m}: A(\xi)=\sum_{i=1}^{N} \xi_{i} A_{i},\right. \\
\left.B(\xi)=\sum_{i=1}^{N} \xi_{i} B_{i}, \sum_{i=1}^{N} \xi_{i}=1, \xi_{i} \geq 0\right\} .
\end{gathered}
$$

Matrices $A_{i}, B_{i}$ and $C$ are known matrices with constant elements and appropriate dimensions.

Consider the following output feedback predictive control algorithm as the controller of NCS

$$
u(t+k)=u(t+k \mid t)=\sum_{j=k}^{N_{u}} F_{k j}[y(t+j \mid t)-w(t+j \mid t)]
$$




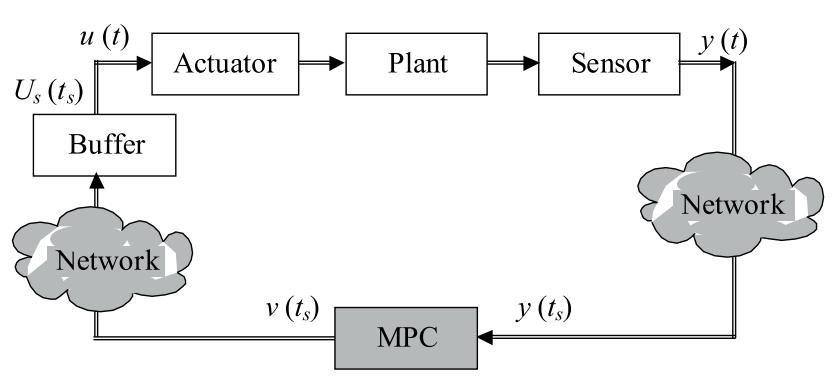

Fig. 1. Configuration of considered networked control system (NCS)

where $k \in\left\{0,1, \ldots, N_{u}-1\right\} ; F_{k j} \in R^{m \times l}$ denotes output feedback gain matrices; $u(t+k \mid t), y(t+k \mid t)$, and $w(t+$ $k \mid t) \in R^{l}$ for $k \geq 1$ denote, respectively, the input, output and desired reference predictions at time instant $t+k$ predicted at time $t(u(t+k \mid t)$ and $x(t+k \mid t)$ are initialized by the measurements of the current state $x(t))$. The prediction is carried out over control horizon $N_{u}$ and prediction horizon $N_{y}=N_{u}$. Input constraints are assumed to be

$$
\left\|u_{i}(t+k)\right\| \leq \bar{u}_{i} ; i=1,2, \ldots, m, k=0,1, \ldots, N_{u}-1
$$

where $\bar{u}_{i}$ is the maximum value of the $i$-th input control $u_{i}(\cdot)$.

Networks exist between sensor and controller and between controller and actuator. It is assumed that in network transmission there is negligible network-induced time delay (time delay is within sampling time of NCS) or it is treated as a dropout $[5,7]$, but packet loss may happen. The sensor and the controller only send data at each sampling time, as well as the controller and actuator receive data. If data are lost at one sampling time, at next sampling time network only transmit new data and old data are discarded. The data are transmitted in a single packet. Based on [27], the packet-loss process in this paper is redefined as follows. Let $\Im=\left\{t_{1}, t_{2}, \ldots, t_{s}, t_{s+1}, \ldots\right\}$ a subsequence of $\{1,2,3, \ldots\}$, denote the sequence of time points of successful data transmissions from the sensor to the actuator, and $l_{p_{-} \max }=\max _{s}\left(t_{s+1}-t_{s}-1\right)$, $s=1,2,3, \ldots ; l_{p \_ \text {max }} \leq N_{u}-1$ be the maximum value of packet-loss number. Then the following concept and mathematical models are introduced to capture the nature of packet losses.

Definition 1. Packet-loss process is defined as

$$
\ell=\left\{\begin{array}{r}
l_{p}\left(t_{s}\right): l_{p}\left(t_{s}\right)=t_{s+1}-t_{s}-1, t_{s} \in \Im \\
0 \leq l_{p}\left(t_{s}\right) \leq l_{p_{-} \max } \leq N_{u}-1
\end{array}\right\}
$$

Definition 2. Packet-loss process (5) is said to be arbitrary if it takes values in $\ell$ arbitrarily from (5).

Note that at time instant $t \in\left\langle t_{s}, t_{s+1}\right\rangle$, if data is not successfully transmitted from the sensor to the controller, the controller will not calculate new control signal for the actuator and as result, the packet-loss occurs.
To overcome the resulting packet-loss problems, we use prediction based compensation schemes from ([2, 5-7]. Instead of a single input, a sequence of predicted future controls $U_{s}\left(t_{s}\right)=\left\{u\left(t_{s}\right), u\left(t_{s}+1\right), \ldots, u\left(t_{s}+l_{p}\right), \ldots, u\left(t_{s}+\right.\right.$ $\left.\left.l_{p_{\text {_max }}}\right)\right\}$ is submitted and implemented at a buffer device with length $l_{p_{\text {_max }}}$ in the actuator. The buffer device is used to store the newest control sequence $U_{s}\left(t_{s}\right)$ transmitted successfully from MPC to actuator at sampling time $t_{s} \in \Im$. At time instant $t \in\left\langle t_{s}, t_{s}+l_{p}\left(t_{s}\right)\right\rangle$, the packet loss occurs, and control action $u(t)$ corresponding to the current sampling time from control sequence $U_{s}\left(t_{s}\right)$ in the buffer device will be applied to the actuator. The main goal of this paper is to design a predictive controller (3) with input constraints (4) so that, control action $u(t)$ from control sequence $U_{s}\left(t_{s}\right)$ robustly stabilizes NCS and ensures input constraints and guaranteed cost of the following cost function (over the infinite optimization horizon) $J=\sum_{t=0}^{\infty} J(t)$, and where

$$
\begin{aligned}
J(t)= & \sum_{k=0}^{N_{y}} x^{\top}(t+k \mid t) Q_{k} x(t+k \mid t)+ \\
& +\sum_{k=0}^{N_{u}-1} u^{\top}(t+k) R_{k} u(t+k),
\end{aligned}
$$

and $Q_{k} \in R^{n \times n}, R_{k} \in R^{m \times m}$ are positive semidefinite (definite) and definite matrices, respectively for all $k\left(Q_{k}=q_{k} I, R_{k}=r_{k} I ; q_{k} \geq 0, r_{k}>0\right)$. and $I-$ is the unitary matrix.

\section{ROBUST MODEL PREDICTIVE CONTROL WITH INPUT CONSTRAINTS}

In this section, we recall some results of the robust MPC design with input constraints from papers [15, 25].

At sampling time $t:=t_{s} \in \Im$, the predicted states of the system (1) for the instant $t+k, k=\left\{0,1, \ldots, N_{u}-1\right\}$ are given by

$$
x(t+k+1 \mid t)=A(\xi) x(t+k \mid t)+B(\xi) u(t+k \mid t) .
$$

Let us define stacked vectors with future states and desired references in corresponding forms as follows

$$
\begin{gathered}
x_{f}(t)=\left[x^{\top}(t) \ldots x^{\top}\left(t+N_{y}-1 \mid t\right)\right]^{\top}, \\
\varpi(t)=\left[w^{\top}(t) \ldots w^{\top}\left(t+N_{y} \mid t\right)\right]^{\top} .
\end{gathered}
$$

Considering $\nu(t)=\left[u^{\top}(t) u^{\top}(t+1) \ldots u^{\top}\left(t+N_{u}-1\right)\right]^{\top}$, state model prediction is obtained as follows

$$
A_{f}(\xi) x_{f}(t+1)=A_{x}(\xi) x(t)+B_{f}(\xi) \nu(t)
$$

where

$$
A_{f}(\xi)=\left[\begin{array}{cccccc}
I & 0 & 0 & \ldots & 0 & 0 \\
-A(\xi) & I & 0 & \ldots & 0 & 0 \\
\vdots & \vdots & \vdots & \ddots & \vdots & \vdots \\
0 & 0 & 0 & \ldots & -A(\xi) & I
\end{array}\right]
$$




$$
\begin{gathered}
\in R^{n N_{y} \times n N_{y}} \\
A_{x}(\xi)=\left[\begin{array}{llll}
A^{\top}(\xi) & 0 & \cdots & 0
\end{array}\right]^{\top} \in R^{n N_{y} \times n} \\
B_{f}(\xi)=\operatorname{diag}\{B(\xi), \ldots, B(\xi)\}^{\top} \in R^{n N_{y} \times m N_{y}}
\end{gathered}
$$

Using definitions of $x_{f}(t)$ and $\varpi(t)$, the predictive control algorithm (3) is also obtained in the following form

$$
\begin{aligned}
\nu(t)=F_{0 x} C x(t)+F_{0 f} C_{f} x_{f} & (t+1)-F_{0} \varpi(t) \\
& =F_{0} C_{m} \eta(t)-F_{0} \varpi(t)
\end{aligned}
$$

where $\eta^{\top}(t)=\left[x^{\top}(t) x_{f}^{\top}(t+1)\right]^{\top}, C_{f}=\operatorname{diag}\{C, \ldots, C\} \in$ $R^{l N_{y} \times n N_{y}}, C_{m}=\operatorname{diag}\left\{C, C_{f}\right\}$ and $F_{0} \in R^{m N_{u} \times l\left(N_{y}+1\right)}$ :

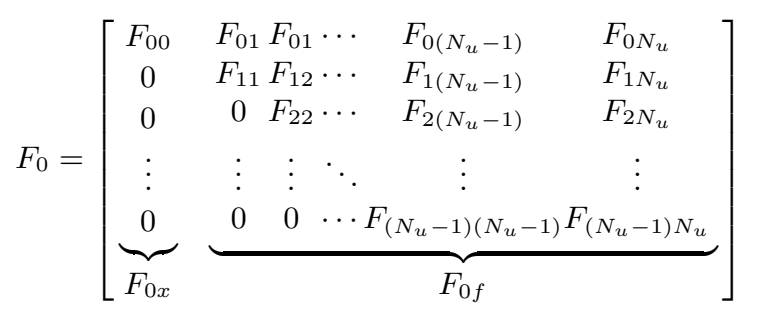

Substituting $\nu(t)$ in the form of (11) into the model prediction (9), the closed-loop model prediction is obtained as follows

$$
A_{c f}(\xi) x_{f}(t+1)=A_{c x}(\xi) x(t)-B_{c f}(\xi) \varpi(t)
$$

where

$$
\begin{aligned}
& A_{c f}(\xi)=A_{f}(\xi)-B_{f}(\xi) F_{0 f} C_{f} \\
& A_{c x}(\xi)=A_{x}(\xi)-B_{f}(\xi) F_{0 x} C \\
& B_{c f}(\xi)=B_{f}(\xi) F_{0}
\end{aligned}
$$

Assuming that the current state $x(t)$ is known, the above equation implies that, the predicted states $x_{f}(t+1)$ are initialized by the current state vector $x(t)$ and known reference signal $\varpi(t)$. Because the vector $\varpi(t)$ is independent on state vector $x(t)$ and if vectors $\varpi(t)$ belong to the class of $\ell_{2}$, then the stability and robustness properties of closed-loop system (13) are not affected by $\varpi(t)$. Therefore, due to Lyapunov function approach, it will be without loss of generality if we set the vector $\varpi(t)$ equal to zero in the case of robust stability analysis or robust controller synthesis.

The cost function (6) can be rewritten as follows

$$
J(t)=\eta^{\top}(t) Q \eta(t)+\nu^{\top}(t) R \nu(t)
$$

where $Q=\operatorname{diag}\left\{Q_{0}, \ldots, Q_{N_{y}}\right\}, R=\left\{R_{0}, \ldots, R_{\left(N_{u}-1\right)}\right\}$.

Associated with the cost function (15), the guaranteed cost control law is defined as follows.

Definition 3. Consider the system (9). If there exists a control law (11) and a positive scalar $J_{0}$ such that for all uncertainties (2), the closed-loop system (13) is asymptotically stable and the closed-loop value of the cost function (15) satisfies $J \leq J_{0}$, then $J_{0}$ is said to be the guaranteed cost and the controller (11) is said to be the guaranteed cost control law.

Finally we recall the well known results from LQ theory.
Lemma 4 [20]. Consider the discrete-time system (9) with control algorithm (11). Control algorithm (11) is the guaranteed cost control law for the closed-loop system (13) if and only if there exists a Lyapunov function $V_{0}(t)=x_{f}^{\top}(t) P_{0}(\xi) x_{f}(t)$ such that the following condition holds

$$
\triangle V_{0}(t)+J(t) \leq 0
$$

Moreover, summarizing (16) from initial time $t_{0}$ to $t \rightarrow \infty$, the following inequality is obtained

$$
-V_{0}\left(t_{0}\right)+J \leq 0
$$

Definition 3 and inequality (17) imply that $J_{0}=V_{0}\left(t_{0}\right)$.

THEOREM. The closed loop system (13) is robustly stable with guaranteed cost $J_{0}$ and parameter dependent quadratic stability if and only if there exist matrices $H_{0} \in R^{n N_{y} \times n\left(N_{y}+1\right)}, P_{0}(\xi)=P_{0}^{\top}(\xi)>0$ and gain matrix $F_{0}$ such that the following bilinear matrix inequality holds [15].

$$
W(\xi) \leq 0
$$

where

$W(\xi)=D(\xi)+A_{m}^{\top}(\xi) H_{0}+H_{0}^{T} A_{m}(\xi)+Q+C_{m}^{T} F_{0}^{T} R F_{0} C_{m}$, $D(\xi)=\operatorname{diag}\left\{-P_{0}(\xi), 0, \ldots, 0, P_{0}(\xi)\right\} \in R^{n\left(N_{y}+1\right) \times n\left(N_{y}+1\right)}$, $A_{m}(\xi)=\left[A_{c x}(\xi)-A_{c f}(\xi)\right] \in R^{n N_{y} \times n\left(N_{y}+1\right)}$.

Consider system (9) where the control $\nu(t)$ is constrained to evolve in the following set

$$
\begin{array}{r}
\Gamma=\left\{\nu(t) \in R^{m N_{u}}:\left\|\nu_{i_{d}}\right\| \leq \bar{u}_{i} ; i_{d}=i+(j-1) m\right. \\
\left.i=1, \ldots, m ; j=1, \ldots, N_{u}\right\}
\end{array}
$$

To derive sufficient stability conditions for input constraints for (13), we consider that the positive invariant region [19], with respect to closed-loop system motion can be defined by the ellipsoidal Lyapunov function set given by $V_{0}(t)$ as follows

$$
\Omega\left(P_{0}(\xi)\right)=\left\{x_{f}(t) \in R^{n N_{y}}: x_{f}^{\top}(t) P_{0}(\xi) x_{f}(t) \leq \theta\right\}
$$

where $\theta$ is a positive real parameter which determines the size of $\Omega\left(P_{0}(\xi)\right)$.

Consider $D_{i_{d}} F_{0}$ denotes the $i_{d}-t h$ row of matrix $F_{0}$

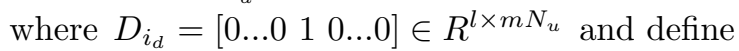

$$
\begin{aligned}
\mathrm{L}\left(F_{0}\right) & =\left\{x_{f}(t) \in R^{n N_{y}}:\left\|D_{i_{d}} F_{0} C_{f} x_{f}(t)\right\| \leq \bar{u}_{i}\right. \\
i d & \left.=i+(j-1) m ; i=1, \ldots, m ; j=1, \ldots, N_{u}\right\}
\end{aligned}
$$

The condition of input constraints reduces to LMI given by the following theorem [25]. 


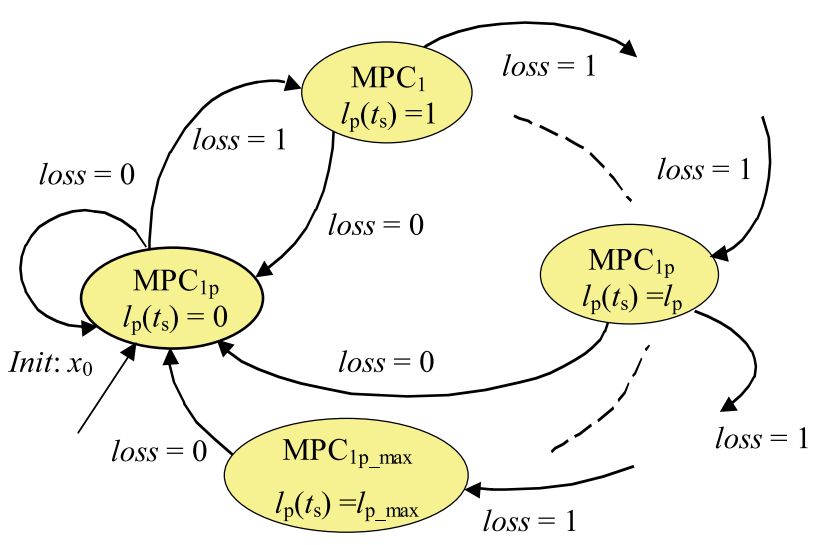

Fig. 2. Schematic representation of a hybrid automaton

TheOREM 6. The inclusion $\Omega\left(P_{0}(\xi)\right) \subseteq L\left(F_{0}\right)$ is for output feedback control equivalent to [25]

$$
\left[\begin{array}{cc}
P_{0}(\xi) & * \\
D_{i_{d}} F_{0} C_{m} & \lambda_{i_{d}}^{0}
\end{array}\right] \geq 0
$$

for all $i_{d}=i+(j-1) m ; i=1, \ldots, m ; j=1, \ldots, N_{u}$ and $\lambda_{i_{d}}^{0} \in\left\langle 0, \frac{\bar{u}_{i}^{2}}{\theta}\right\rangle$.

Without regard to the networks (the packet-loss problem is not considered in the controller design), if conditions (18) and (22) hold, then guaranteed MPC controller (3) is robustly stabilizes uncertain system (1) and guarantees input constraints (4). To fix the packet-loss problem, the compensation mechanism introduced in Section 2 is used. The first $l_{p_{-} \max }$ elements of vector $\nu(t)$ are encapsulated into one packet so-called control sequence $U_{s}(t)=\left\{u\left(t_{s}\right), u\left(t_{s}+1\right), \ldots, u\left(t_{s}+l_{p}\right), \ldots, u\left(t_{s}+l_{p_{-} \max }\right)\right\}$ and sent to the buffer device in the actuator. However, control sequence $U_{s}\left(t_{s}\right)$ obtained from the above MPC controller design(without regard to the networks) can induce instability or poor performance (the guaranteed cost and the input constraints) of the NCS. This problem will be solved in the next section.

\section{ROBUST NETWORKED MODEL PREDICTIVE CONTROL DESIGN}

In this section, a necessary and sufficient robust stability conditions to robust networked MPC design with guaranteed cost control law for the case of lost packet are presented. Main result of this section on robust networked MPC design can be summarized in Theorem 8 .

Due to the compensation mechanism, the control action $u(t)$ corresponding to the current sampling time from control sequence $U_{s}\left(t_{s}\right)$ in the buffer device will be applied to the actuator at every sampling time $t \in$ $\left\langle t_{s} ; t_{s}+l_{p}\left(t_{s}\right)\right\rangle\left(l_{p}\left(t_{s}\right)\right.$ packets are lost). This process is considered as a switched system with schematic representation of a hybrid automaton in Fig. 2. Hybrid state of the hybrid automat is $x_{h}(t)=\left(l_{p}(t), x_{f}(t)\right)$ in which number packet loss $l_{p}(t) \in \ell$ is a discrete state, $x_{f}(t)$ is the state of continuous part. Activity function is defined by closed-loop output feedback model predictive controls $M P C_{l_{p}}$ in (29).

At sampling time $t:=t_{s}\left(l_{p}=0\right)$, model predictive control $M P C_{l_{p}}$ defined in Section 2 is used to compute control sequence $U_{s}(t)$. If no packet loss at $t+1, M P C_{0}$ is applied. Otherwise, jump to $M P C_{1}$, it means that one packet lost.

Generally, at sampling time $t:=t_{s}+l_{p} \quad\left(1 \leq l_{p} \leq\right.$ $\left.l_{p_{\_} \max }\right), M P C_{l_{p}}$ is applied. If no packet loss at $t+1$, jump to $M P C_{0}$. Otherwise, if $l_{p}<l_{p_{-} \max }$, jump to $M P C_{l_{p}+1}$ and it means that $l_{p}+1$ packets are lost. The predicted states of $M P C_{l_{p}}$ for the instant $t+k, k=0, \ldots, N_{u}-1$, are given by

$$
\begin{aligned}
& x(t+k+1 \mid t)=A(\xi) x(t+k \mid t)+B(\xi) u(k), \\
& u(k)=\left\{\begin{array}{l}
u\left(t_{s}+l_{p}+k\right) \quad \text { if } 0 \leq k \leq N_{u}-l_{p}-1, \\
u\left(t_{s}+N_{u}-1\right) \text { if } N_{u}-l_{p} \leq k \leq N_{u}-1 .
\end{array}\right.
\end{aligned}
$$

Based on equation (3), $u\left(t_{s}+l_{p}+k\right)$ for $0 \leq k \leq N_{u}-l_{p}-1$ can be rewritten as follows

$$
u\left(t_{s}+l_{p}+k\right)=\sum_{j=l_{p}+k}^{N_{u}} F_{\left(l_{p}+k\right) j} C x\left(t_{s}+j \mid t_{s}\right) .
$$

Substituting $j$ by $i+l_{p}$ to $(24)$, we obtain

$$
u\left(t_{s}+l_{p}+k\right)=\sum_{i=k}^{N_{u}-l_{p}} F_{\left(l_{p}+k\right)\left(l_{p}+i\right)} C x\left(t_{s}+l_{p}+i \mid t_{s}\right)
$$

for $0 \leq k \leq N_{u}-l_{p}-1$.

If the following condition

$$
x\left(t_{s}+l_{p}+i \mid t_{s}\right)=x\left(t_{s}+l_{p}+i \mid t_{s}+l_{p}\right)=x(t+i \mid t)
$$

holds for $0 \leq i \leq N_{u}-l_{p}$, then the state model of $M P C_{l_{p}}$ is obtained in the form of (9) with the following control algorithm

$$
\nu\left(t, l_{p}\right)=F_{l_{p}} C_{m} \eta(t)=F_{l_{p} x} C x(t)+F_{l_{p} f} C_{f} x_{f}(t+1)
$$

where $F_{l_{p}}$ is created by rearrange elements of gain matrix $F_{0}$ and has the following structure

$$
\begin{aligned}
& F_{l_{p}}=
\end{aligned}
$$

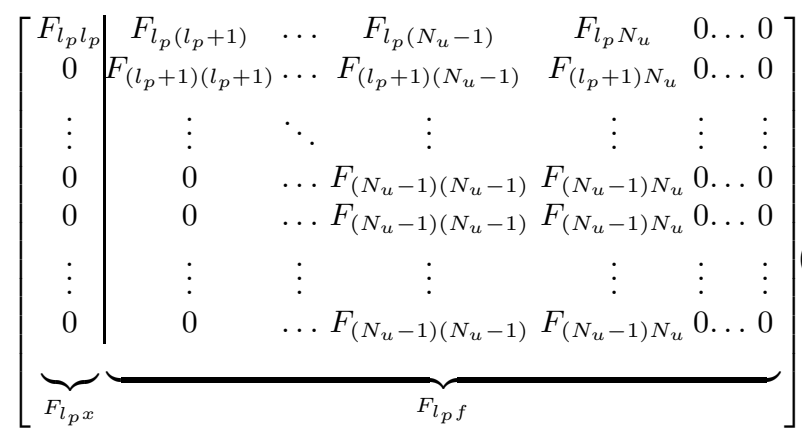


According to mathematical induction (a method of mathematical proof), condition (26) holds for all $1 \leq$ $l_{p} \leq l_{p_{-} \text {max }}$, if we prove that, (26) holds for the cases of $l_{p}=\{1,2\}$, and with assumption that (26) holds for $l_{p}-1$, it will hold for $l_{p}$.

Due to the result of solving difference equation (1), $x\left(t_{s}+1 \mid t_{s}\right)=x\left(t_{s}+1 \mid t_{s}+1\right)$ holds for $i=0$. Using (7) and (23) for $i=1$, we obtain $x\left(t_{s}+2 \mid t_{s}\right)=x\left(t_{s}+2 \mid t_{s}+1\right)=$ $A(\xi) x\left(t_{s}+1 \mid t_{s}\right)+B(\xi) u\left(t_{s}+1\right)$. Sequentially repeating the previous step for $i=2, \ldots, N_{u}-1$, it is easily to show that $x\left(t_{s}+i+1\right)=x\left(t_{s}+i+1 \mid t_{s}+1\right)$. Condition (26) holds for $l_{p}=1$.

Repeating all steps and using results in the above proof for $l_{p}=1$, we can prove that, condition (26) holds for $l_{p}=2$.

Now, (26) is supposed to hold for $l_{p}-1$. We have to show that, (26) will hold for $l_{p}$. Indeed, for $i=0$, we have $x\left(t_{s}+l_{p} \mid t_{s}+l_{p}\right)=x\left(t_{s}+l_{p} \mid t_{s}+l_{p}-1\right)=A(\xi) x\left(t_{s}+\right.$ $\left.l_{p}-1 \mid t_{s}+l_{p}-1\right)+B(\xi) u\left(t_{s}+l_{p}-1\right)$. Because (26) holds for $l_{p}-1$, then $x\left(t_{s}+l_{p}-1 \mid t_{s}+l_{p}-1\right)=x\left(t_{s}+l_{p}-1 \mid t_{s}\right)$. As a result, $x\left(t_{s}+l_{p} \mid t_{s}\right)=x\left(t_{s}+l_{p} \mid t_{s}+l_{p}\right)$. By the same way for $i=\left\{1, \ldots, N_{u}-l_{p}-1\right\},(26)$ is true for $l_{p}$. The proof of (26) is obtained.

Applying control algorithm (27) into model prediction (9), the closed-loop MPC of $M P C_{l_{p}}$ is obtained as follows

$$
A_{c f}\left(\xi, l_{p}\right) x_{f}(t+1)=A_{c x}\left(\xi, l_{p}\right) x(t)
$$

where

$$
\begin{aligned}
& A_{c f}\left(\xi, l_{p}\right)=A_{f}(\xi)-B_{f}(\xi) F_{l_{p} f} C_{f}, \\
& A_{c x}\left(\xi, l_{p}\right)=A_{x}(\xi)+B_{f}(\xi) F_{l_{p} x} C .
\end{aligned}
$$

Let us define the following parameter-dependent Lyapunov functional candidate for the closed-loop feedback of $M P C_{l_{p}}$.

$$
\begin{aligned}
& V_{l_{p}}(t)=x_{f}^{\top}(t) \bar{P}_{l_{p}}(\xi) x_{f}(t), \\
& \bar{P}_{l_{p}}(\xi) \in R^{n N_{y} \times n N_{y}}: \bar{P}_{l_{p}}(\xi)=\operatorname{diag}\left\{P_{l_{p}}(\xi), \ldots, P_{l_{p}}(\xi)\right\} \\
& P_{l_{p}}(\xi) \in R^{n \times n}: P_{l_{p}}(\xi)=\sum_{i=1}^{N} \xi_{i} P_{l_{p} i} ; P_{l_{p} i}=P_{l_{p}}^{\top}>0, \\
& \xi_{i} \geq 0, \sum_{i=1}^{N} \xi_{i}=1 .
\end{aligned}
$$

The cost function for closed-loop feedback for NCS is rewritten as the follows.

$$
\begin{gathered}
J(t)=x^{\top}(t) Q_{0} x(t)+x_{f}^{\top}(t+1) Q_{f} x_{f}(t+1)+ \\
\left(F_{l_{p} x} C x(t)+F_{l_{p}} C_{f} x_{f}(t+1)\right)^{\top} R \times \\
\left(F_{l_{p} x} C x(t)+F_{l_{p} f} C_{f} x_{f}(t+1)\right)= \\
\eta^{\top}(t)\left(Q+C_{m}^{\top} F_{l_{p}}^{\top} R F_{l_{p}} C_{m}\right) \eta(t)
\end{gathered}
$$

where $Q_{f}=\operatorname{diag}\left\{Q_{1}, Q_{2}, \ldots, Q_{N_{y}}\right\}$.

Applying theory of switched system for discrete system $[11$, p.129] and using Lemma 4 , the switched system with the schematic representation of hybrid automaton in Fig. 2 is robust stable with guaranteed cost if and only if the following conditions hold

$$
\begin{gathered}
x_{f}^{\top}(t+1) \bar{P}_{i_{p}} x_{f}(t+1)-x_{f}^{\top}(t) \bar{P}_{l_{p}} x_{f}(t)+J(t) \leq 0, \\
i_{p}=\left\{0, l_{p}+1\right\}
\end{gathered}
$$

or

$$
\begin{gathered}
J(t)-x^{\top}(t) P_{l_{p}} x(t)+x^{\top}\left(t+N_{y} \mid t\right) P_{i_{p}} x\left(t+N_{y} \mid t\right)+ \\
+\sum_{k=1}^{N_{y}-1} x^{\top}(t+k \mid t)\left(P_{i_{p}}(\xi)-P_{l_{p}}(\xi)\right) x(t+k \mid t) \leq 0 \\
i_{p}=\left\{0, l_{p}+1\right\}
\end{gathered}
$$

Note the switched control algorithm for the case of lost packet is given in Fig. 2.

Necessary and sufficient condition for robust stability with guaranteed cost for the NCS with packet loss is given by the following Lemma.

Lemma 7. Control sequence $U_{s}\left(t_{s}\right)$ is the guaranteed cost control law for the NCS with packet loss if and only if the following condition holds

$$
\begin{aligned}
B_{l_{p}}^{i_{p}}(\xi)= & A_{c}^{\top}\left(\xi, l_{p}\right)\left(\widetilde{P}_{l_{p}}^{i_{p}}(\xi)+Q_{f}\right) A_{c}\left(\xi, l_{p}\right)-P_{l_{p}}(\xi)+ \\
+Q_{0}+ & \left(F_{l_{p} x} C+F_{l_{p} f} C_{f} A_{c}\left(\xi, l_{p}\right)\right)^{T} R \times \\
& \times\left(F_{l_{p} x} C+F_{l_{p} f} C_{f} A_{c}\left(\xi, l_{p}\right)\right) \leq 0
\end{aligned}
$$

where $i_{p}=\left\{0, l_{p}+1\right\}, \widetilde{P}_{l_{p}}^{i_{p}}(\xi) \in R^{n N_{y} \times n N_{y}}$ and

$$
\widetilde{P}_{l_{p}}^{i_{p}}(\xi)=\operatorname{diag}\left\{P_{i_{p}}-P_{l_{p}}, \ldots, P_{i_{p}}-P_{l_{p}}, P_{i_{p}}\right\}(\xi) .
$$

Robust stability condition (35) is not directly applicable due to its numerical complexity. In the following theorem the novel formulation of robust stability condition is developed, which provide LMI for MPC robust stability analysis and BMI for MPC robust design.

THEOREM 8. Control sequence $U_{s}\left(t_{s}\right)$ robustly stabilizes the NCS with loss packet process $\ell$ and ensures the guaranteed cost $J_{0}$, input constraints (4) if and only if there exist matrices $H_{l_{p}} \in R^{n N_{y} \times n\left(N_{y}+1\right)}, P_{l_{p}}(\xi)=P_{l_{p}}^{\top}(\xi)>$ 0 , and gain matrices $F_{l_{p}}$ such that the following bilinear matrix inequality $(B M I)$

$$
\begin{gathered}
W_{l_{p}}^{i_{p}}(\xi)=D_{l_{p}}^{i_{p}}(\xi)+A_{m}^{\top}\left(\xi, l_{p}\right) H_{l_{p}}^{i_{p}}+H_{l_{p}}^{i_{p}} A_{m}\left(\xi, l_{p}\right)+ \\
+Q+C_{m}^{\top} F_{l_{p}}^{\top} R F_{l_{p}} C_{m} \leq 0 \\
i_{p}=\left\{0, l_{p}+1\right\}
\end{gathered}
$$

and the following linear matrix inequality (LMI)

$$
\begin{gathered}
{\left[\begin{array}{cc}
P_{l_{p}}(\xi) & * \\
D_{i_{d}} F_{l_{p}} C_{m} & \lambda_{i_{d}}^{l_{p}}
\end{array}\right] \geq 0 ; \lambda_{i_{d}}^{l_{p}} \in\left\langle 0, \frac{\bar{u}_{i}^{2}}{\theta}\right\rangle ;} \\
i_{d}=i+(j-1) m, i=1, \ldots, m \\
j=1, \ldots, N_{u}-l_{p}
\end{gathered}
$$


hold for all $0 \leq l_{p} \leq l_{p \_ \text {max }}$.

Where $D_{l_{p}}^{i_{p}} \in R^{n\left(N_{y}+1\right) \times n\left(N_{y}+1\right)}$ and

$$
\begin{aligned}
& D_{l_{p}}^{i_{p}}(\xi)=\operatorname{diag}\left\{-P_{l_{p}}, P_{i_{p}}-P_{l_{p}}, \ldots, P_{i_{p}}-P_{l_{p}}, P_{i_{p}}\right\}(\xi), \\
& A_{m}\left(\xi, l_{p}\right)=\left[A_{c x}\left(\xi, l_{p}\right)-A_{c f}\left(\xi, l_{p}\right)\right]
\end{aligned}
$$

Pr o of . Sufficiency. Considering $H_{l_{p}}^{i_{p}}=\left[H_{l_{p} x}^{i_{p}} H_{l_{p} f}^{i_{p}}\right]$, where $H_{l_{p} x}^{i_{p}} \in R^{n N_{y} \times n}$ and $H_{l_{p} f}^{i_{p}} \in R^{n N_{y} \times n N_{y}}$, the inequality (37) can be rewritten as

$$
W_{l_{p}}^{i_{p}}(\xi)=\left[\begin{array}{ll}
W_{11}(\xi) & W_{12}(\xi) \\
W_{12}^{\top}(\xi) & W_{22}(\xi)
\end{array}\right] \leq 0
$$

where

$W_{11}(\xi)=-P_{l_{p}}(\xi)+H_{l_{p} x}^{i_{p}} A_{c x}\left(\xi, l_{p}\right)+A_{c x}^{\top}\left(\xi, l_{p}\right) H_{l_{p} x}^{i_{p}}+$ $Q_{0}+C^{\top} F_{l_{p} x}^{\top} R F_{l_{p} x} C, W_{12}(\xi)=-H_{l_{p} x}^{i_{p}}{ }^{\top} A_{c f}\left(\xi, l_{p}\right)+$ $A_{c x}^{\top}\left(\xi, l_{p}\right) H_{l_{p} f}^{i_{p}}+C^{T} F_{l_{p} x}^{\top} R F_{l_{p} f} C_{f}$, and $W_{22}(\xi)=\widetilde{P}_{l_{p}}^{i_{p}}(\xi)-$ $H_{l_{p} f}^{i_{p}}{ }^{\top} A_{c f}\left(\xi, l_{p}\right)+A_{c f}^{\top}\left(\xi, l_{p}\right) H_{l_{p} f}^{i_{p}}+Q_{f}+C_{f}^{T} F_{l_{p} f}^{\top} R F_{l_{p} f} C_{f}$.

Since the matrix $L=\left[\begin{array}{ll}I & A_{c}^{\top}\left(\xi, l_{p}\right)\end{array}\right]$ has full row rank, multiplying the left of (40) by $L$ and the right by $L^{\top},(35)$ is obtained. It means that, the sufficiency is proved.

Necessity. Suppose that there exist symmetric positive definite matrices $P_{l_{p}}(\xi)$ and $P_{i_{p}}(\xi)$ such that robust stability condition (35) holds; necessarily, there exists a scalar $\beta_{l_{p}}^{i_{p}}>0$ such that

$$
A_{c}^{\top}\left(\xi, l_{p}\right)\left(\widetilde{P}_{l_{p}}^{i_{p}}(\xi)+\beta_{l_{p}}^{i_{p}} I\right) A_{c}\left(\xi, l_{p}\right)-P_{l_{p}}(\xi) \leq 0 .
$$

Applying Schur complement formula to (41), we obtain

$$
\left.\left[\begin{array}{cc}
-P_{l_{p}}(\xi) & A_{c}^{\top}\left(\xi, l_{p}\right)\left(\widetilde{P}_{l_{p}}^{i_{p}}(\xi)+\beta_{l_{p}}^{i_{p}} I\right) \\
* & -\left(\widetilde{P}_{l_{p}}^{i_{p}}(\xi)+\beta_{l_{p}}^{i_{p}} I\right)
\end{array}\right]\right] \leq 0
$$

Taking $H_{l_{p} x}=-\frac{1}{2} \beta_{l_{p}}^{i_{p}}\left(A_{c f}^{-1}\left(\xi, l_{p}\right)\right)^{\top} A_{c}\left(\xi, l_{p}\right)$ and $H_{l_{p} f}=$ $\left(A_{c f}^{-1}\left(\xi, l_{p}\right)\right)^{\top}\left(\widetilde{P}_{l_{p}}^{i_{p}}(\xi)+\frac{1}{2} \beta_{l_{p}}^{i_{p}} I\right)$, after some manipulations the following inequality is obtained.

$$
\left[\begin{array}{ll}
W_{11 \beta}(\xi) & W_{12 \beta}(\xi) \\
W_{12 \beta}^{\top}(\xi) & W_{22 \beta}(\xi)
\end{array}\right] \leq 0
$$

where $W_{12 \beta}(\xi)=W_{12}(\xi), W_{22 \beta}(\xi)=W_{22}(\xi)$ and $W_{11 \beta}(\xi)=W_{11}(\xi)+\beta_{l_{p}}^{i_{p}} A_{c}^{\top}\left(\xi, l_{p}\right) A_{c}\left(\xi, l_{p}\right)$.

Because $\beta_{l_{p}}^{i_{p}} A_{c}^{\top}\left(\xi, l_{p}\right) A_{c}\left(\xi, l_{p}\right) \geq 0$, then the inequality (40) resp. the inequality (37) is obtained which proves the necessity. For guaranteed cost the proof goes the analogical way as given above.

To prove condition (38) for input constraints, see [25]. Theorem 8 is proved.

Note that $(37)$ is affine to $\xi$. If $W_{l_{p} j}^{i_{p}} \leq 0, j=$ $1, \ldots, N$, is feasible with respect to unknown $P_{l_{p} j}=$
$P_{l_{p} j}^{\top}>0, P_{i_{p} j}=P_{i_{p} j}^{\top}>0, H_{l_{p}}^{i_{p}}$, and $F_{l_{p}}$ for all $0 \leq l_{p} \leq l_{p_{-} \max }, i_{p}=\left\{0, l_{p}+1\right\}$, then the control sequence $U_{s}\left(t_{s}\right)$ guarantees robust stability and guaranteed cost for NCS with predictive control (3) within the convex set defined by (2). Therefore, BMI robust stability condition "if and only if" in (37) reduces to sufficient condition.

\section{EXAMPLES}

In this section, we present the results of numerical calculations and simulations for a numerical example to demonstrate the effectiveness of the proposed method, namely its ability to cope with robust stability, guaranteed cost, and input constraints without complex computational load. Numerical calculations have been realized by using PEN-BMI.

The discrete model of double integrator turns to (1) where

$$
A_{0}=\left[\begin{array}{ll}
1 & 0 \\
1 & 1
\end{array}\right], B_{0}=\left[\begin{array}{l}
1 \\
0
\end{array}\right], C=\left[\begin{array}{ll}
1 & 0
\end{array}\right]
$$

and uncertainty matrices are

$$
A_{1 u}=\left[\begin{array}{ll}
0.01 & 0.01 \\
0.02 & 0.03
\end{array}\right], \quad B_{1 u}=\left[\begin{array}{c}
0.001 \\
0
\end{array}\right]
$$

For the case when number of uncertainty is $p=1$ the number of vertices is $N=2^{p}=2$, the matrices (2) corresponding to two working points 1.wp and 2.wp are calculated as follows

$$
\left[\begin{array}{ll}
A_{1}=A_{0}+A_{1 u} & B_{1}=B_{0}+B_{1 u} \\
A_{2}=A_{0}-A_{1 u} & B_{2}=B_{0}-B_{1 u}
\end{array}\right]
$$

Considering with prediction horizon and control horizon as $N_{u}=N_{y}=8$. We assume that the packet-loss upper bound $l_{p_{-} \max }=N_{u}-1=7$, which means that up to $87.5 \%$ of the packets, can be lost during the network transmissions.

Applying Theorem 8 with parameters $\theta=30$ and $\bar{u}_{i}=1$ for the input constraints, and $(Q=q I ; R=r I)$

$q=\left\{q_{i}\right\}_{i=0}^{8}=\{0.001 ; 0.0025 ; 0.005 ; 0.0075 ; 0.01 ; 0.01 ;$ $0.015 ; 0.075 ; 0.1\}$,

$r=\left\{r_{i}\right\}_{i=0}^{7}=\left\{1 ; 10 ; 100 ; 1000 ; 10^{4} ; 10^{5} ; 10^{6} ; 10^{7}\right\}$ for the cost function, the gain matrix $F_{0}$ of predictive control algorithm (3) is obtained as follows

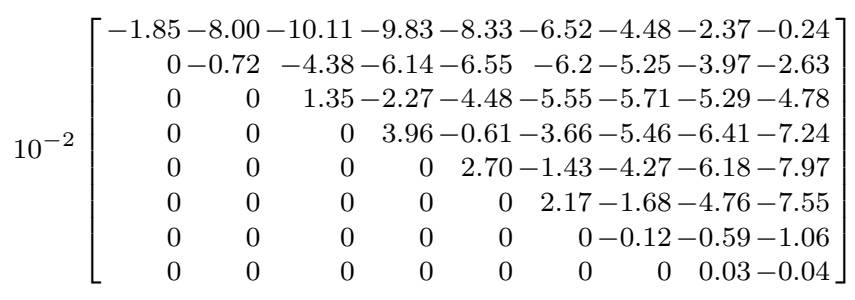




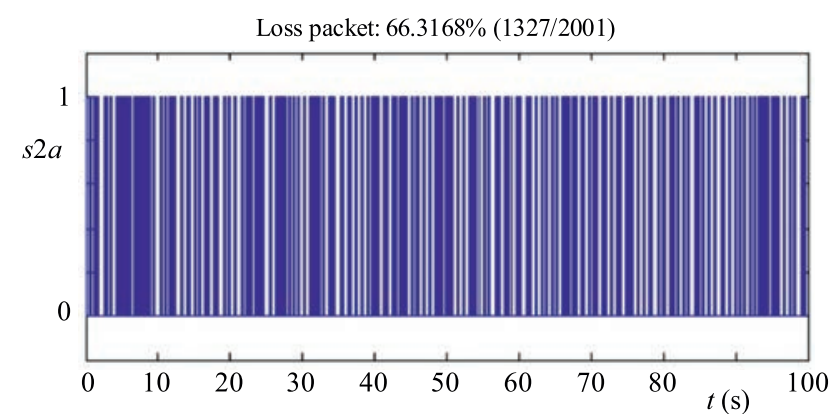

Fig. 3. The status of data transmission in the network
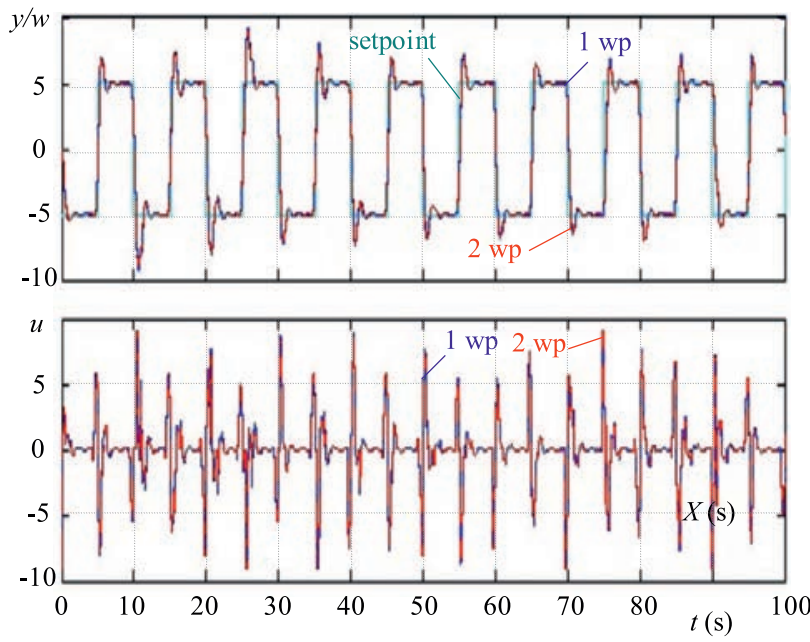

Fig. 4. Simulations of output and input control signals at two operating points
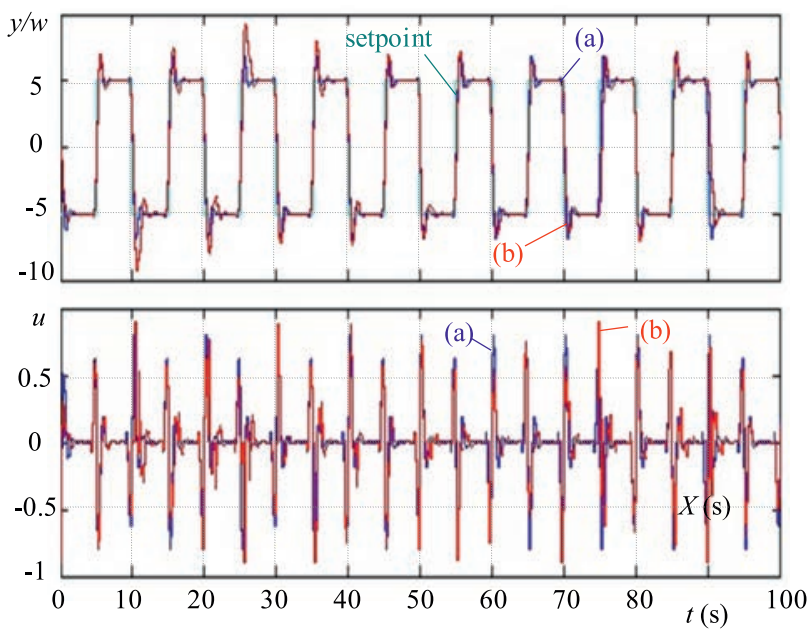

Fig. 5. Comparison between the case of packet loss and no packet loss in the network: (a) - no loss packet, (b) - loss packet

Our simulations are based on the framework in Fig. 1. The status of data transmission in the network is shown in Fig. 3, where $s 2 a=1(s 2 a=0)$ indicates that the data is (is not) successfully sent from the sensor to the actuator. We can see that, only 1327 of 2001 packets were successfully transmitted via network; it means that $66.318 \%$ of packets were lost. Fig. 4 shows simulations of output and input control signals at two operating points 1.wp and 2.wp. It shows that networked MPC is robustly stable and guarantees input constraints. In Fig. 5, there is comparison between the case of packet loss and no packet loss in the network at the first operating point. Comparison between the results obtained by design method without considering packet loss and proposed method at the first operating point is shown in Fig. 6. It shows that, the design method without considering packet also stabilizes NCS, but gives less performance than the proposed method.
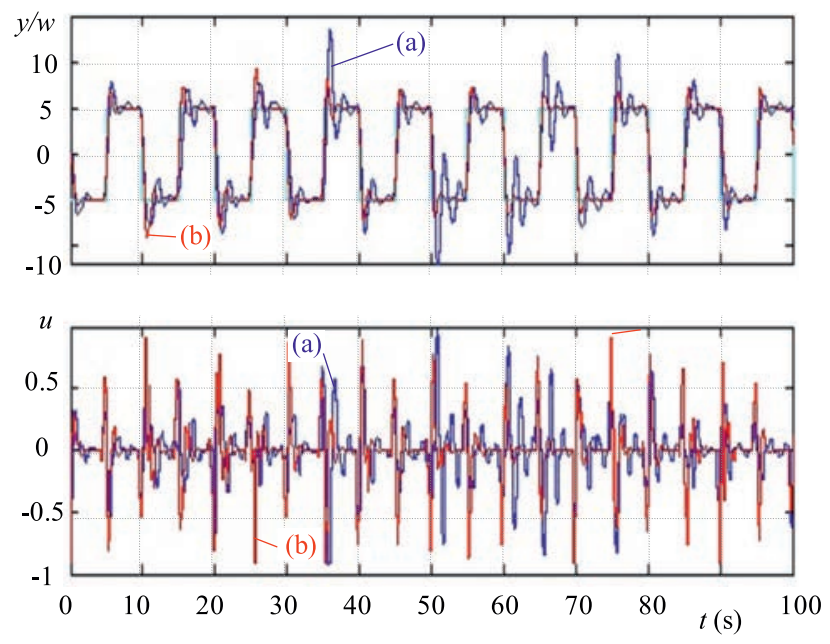

Fig. 6. Comparison between the case of design method without considering packet loss and the proposed method: (a) - without considering packet loss, (b) - considering packet loss

\section{CONCLUSION}

The stabilization of networked predictive control system with packet-loss was studied in this paper. The packet-loss process is arbitrary and bounded by the control horizon of model predictive control. Networked predictive control systems with packet loss are modeled as switched linear systems. This enables us to apply the theory of switched systems to establish the stability condition of networked predictive control systems. The stabilizing controller design is based on sufficient robust stability conditions formulated as a solution of bilinear matrix inequality BMI. The effectiveness of the proposed method was illustrated by a numerical example and simulations.

\section{Acknowledgements}

The work has been supported by Grant 1/1241/12 of the Slovak Scientific Grant Agency.

\section{REFERENCES}

[1] AZIMI-SADJADI, B. : Stability of Networked Control Systems in the Presence of Packet Losses, In: Proceedings of the Conference on Decision and Control, Hawaii, USA, 2003, pp. 676-681.

[2] CHEN, J.-IRWIN-G. W.-McKERNAN, A. : Packet-Based Robust mpc for Wireless Networked Control using Co-Design, In: American Control Conference, Marriott Waterfront, Baltimore, MD, USA, 2010, pp. 1829-1834.

[3] DING, B.: A Synthesis Approach of Model Predictive Control for Linear Systems over Networks with Bounded Packet Loss, 
In: Proceedings of the 8th IEEE International Conference on Control and Automation, Xiamen, China, 2010, pp. 2258-2263.

[4] DING, B.: Stabilization of Linear Systems over Networks with Bounded Packet Loss and its Use in Synthesizing Model Predictive Control, In: Proceedings of 8th International Conference on Control and Automation, Xiamen, China, pp. 2258-2263.

[5] FINDEISEN, R.-GRÜNE, L.-PANNEK, J.-VARUTTI, P. : Robustness of Prediction Based Delay Compensation for Nonlinear Systems, In: Proceedings of the 18th IFAC World Congress, Milano, Italy, pp. 203-208.

[6] GRÜNE, L.-PANNEK, J.-WORTHMANN, K. : A Networked Unconstrained Nonlinear mpc Scheme, In: Proceedings of the European Control Conference, 2009, pp. 91-96.

[7] GRÜNE, L.-PANNEK, J.-WORTHMANN, K. : A Prediction Based Control Scheme for Networked Systems with Delays and Packet Dropouts, In: Proceedings of the 48th IEEE CDC, Shanghai, China, 2009, pp. 537-542.

[8] LEE, J. H.-COOLEY, B.: Recent advances in model predictive control, Chemical Process Control 93 No. 316 (2007), 201-216, AIChE Symposium Series - American Institute of Chemical Engineers.

[9] LI, Z. J. SUN, D. H.-SHI, Y. T.-WANG, L. F. : A Stabilizing Model Predictive Control for Network Control System with Data Packet Dropout, Journal of Control Theory and Application 7 No. 3 (2009), 281-284.

[10] LIU, G. P.-MU, J. X.-REES, D.: Networked Predictive Control of Systems with Random Communication Delay, In: UKACC 2004 International Conference on Control, Bath, UK, 2004, ID-015

[11] LUNZE, J.-LAMNABHI-LAGARRIGUE, F. : Handbook of Hybrid Systems Control, Cambridge University Press, The Edinburgh Building, Cambridge CB2 8RU, UK, 2009.

[12] MU, J. X.-LIU, G. P.-REES, D. : Design of Robust Networked Predictive Control Systems, In: American Control Conference, vol. 1, 2005, pp. 638-643.

[13] NEŠIÍC, D.-TEEL, A. R. : Input-to-State Stability of Networked Control Systems, Automatica 40 No. 12 (2004), 2121-2128.

[14] NGUYEN, Q. T.-VESELÝ, V.: Design of Robust Networked Predictive Control Systems with Packed Loss, In: 4th IFAC NMPC'12, August, The Netherlands, 2012, CD-ROM.

[15] NGUYEN, Q. T.-VESELÝ, V.-ROSINOVÁ, D. : Design of Robust Model Predictive Control with Input Constraints, International Journal of Systems Science (2011), http://dx.doi.org/10.1080/00207721.2011.627476.

[16] NILSON, J.: Real-time Control System with Delays, Department of Automatic Control, Lund Institute of Technology, Lund, Sweden, 1998.

[17] QIN, S. J.-BADGEWELL, T. : An Overview of Industrial Model Predictive Control Technology, Chemical Process Control 93 No. 316 (1997), 232-256, AIChe Symposium Series American Institute of Chemical Engineers.

[18] RAY, A.-HALEVY, Y.: Integrated Communication and Control Systems: Part ii Design Considerations, ASME Journal of Dynamic Systems, Measurement and Control 110 (1988), 374-381.

[19] ROHAL-ILKIV, B.: A Note on Calculation of Polytopic Invariant and Feasible Sets for Linear Continuous-Time Systems, Annual Review in Control 28 (2004), 59-64.

[20] ROSINOVÁ, D.-VESELÝ, V.-KUČERA, V.: A necessary and Sufficient Condition for Static Output Feedback Stabilizability of Linear Discrete-Time Systems, Kybernetika 39 No. 4 (2003), 447-459.

[21] SEILER, P.-SENGUPTA, R. : An $h_{\infty}$ Approach to Networked Control, IEEE Transactions on Automatic Control 50 (2005), 356-364.
[22] SRINIVASAGUTA, D.-SCHATTLER, H.-JOSEPH, B. : Time-Stamped Model Predictive Control of Processes with Random Delays, Computer and Chemical Engineering 28 No. 3 (2004), 1337-1346.

23] TANG, P. L.-de SILVA, C. W.: Ethernet-Based Predictive Control for an Industrial Hydraulic Machine, In: Proceedings of IEEE Conference on Decision and Control, vol. 1, 2003, pp. 695-700.

24] TANG, P. L.- de SILVA, C. W.: Stability and Optimality of Constrained Model Predictive Control with Future Input Buffering in networked Control Systems, In: American Control Conference, vol. 2, 2005, pp. 1245-1250.

[25] VESELÝ, V.-ROSINOVÁ, D.-FOLTIN, M. : Robust Model Predictive Control Design with Input Constraints, ISA Transactions 49 (2010), 114-12.

[26] WALSH, G. C.-YE, H.-BUSHNELL, L.: Stability Analysis of Networked Control Systems, In: Proceedings of the American Control Conference, 1999, pp. 2876-2880.

27] XIONG, J.-LAM, J.: Stabilization of Linear Systems over Networks with Bounded Packet Loss, Automatica 43 (2007), 80-87.

28] YU, M.-WANG, L.-CHU, T.-XIE, G. : Stabilization of Networked Control Systems with Data Packet Dropout and Network Delays via Switching System Approach, In: 43rd IEEE conference on decision and control, Atlantis, Paradise Island, ahamas, 2004, pp. 3539-3544.

29] ZHANG, W.-BRANICKY, M. S.-PHILIP, S. M. : Stability of Networked Control Systems, IEEE Control System Magazine 21 No. 1 (2001), 84-89.

Received 14 April 2013

Quang Thuan Nguyen was born in Hanoi, Vietnam. He received the Master degree in Cybernetics from Slovak University of Technology in Bratislava, Faculty of Electrical Engineering and Information Technology in and $\mathrm{PhD}$ degree in 2012 in field of robust control, model predictive control and network control system. Now, he works as a programmer for TV smart hub applications at Samsung Electronics Slovakia (SEKS).

Vojtech Veselý (Prof, Ing, DrSc) was born in 1940. Since 1964 he has worked at the Department of Automatic Control Systems at the Faculty of Electrical Engineering and Information Technology, Slovak University of Technology in Bratislava. Since 1986 he has been Full Professor. His research interests include the areas of power system control, decentralized control of large-scale systems, process control and optimization.

Alena Kozáková (Assoc Prof, Ing, PhD) graduated from the Faculty of Electrical Engineering and Information Technology, Slovak University of Technology (FEI STU) in 1985 and received her PhD in Technical Cybernetics in 1998. Since 1985 she has been with the Department of Automatic Control Systems, FEI STU. Within her research and teaching activities she focuses on robust and optimal control.

Pavel Pakshin (Prof, DrSc), was born in 1951. In 1993 he has worked at the Arzamas Polytechnic Institute of R.E. Alekseev Nizhny Novgorod State Technical University where he is the Head of Department of Applied Mathematics since 1998. Professor Pakshin is associate editor of International Journal of Innovational Computing and Information Control (IJICIC), ICIC Express Letters and Numerical Analysis, Control and Optimization (NACO), his current research interests are in the field of stochastic and robust stability and control. 